\title{
Redundant Inertial-Aided GBAS for Civil Aviation
}

\author{
Anja Grosch, Boubeker Belabbas and Michael Meurer \\ German Aerospace Center \\ Institute of Communications and Navigation \\ Oberpfaffenhofen, Germany
}

\begin{abstract}
In this article, we investigate the impact of redundant MEMS-IMU on a loosely coupled INS/GBAS system. Therefore, we consider and analyze the combined performance of three identically and orthogonally mounted low-cost IMUs. It is shown that due to the redundancy, the INS based position error is reduced by an average factor of two. In conventional GBAS, no position solution can be obtained while GPS is no available which lead to a full system failure. It is demonstrated that our redundant INS/GBAS integration sufficiently bridges GPS outages up to 10 seconds. Hence, the system availability is significantly improved. Additionally, an integrity concept is introduced providing integrity information even in the case where no GPS solution is available. This is essential for all safety-of-life applications such as civil aviation. We analyze our concept both, analytically and by Monte-Carlo Simulations. Our investigations clearly show that the robustness of the GBAS can be dramatically increased while maintaining low-costs and low-complexity.
\end{abstract}

\section{INTRODUCTION}

Low cost Inertial Measurement Units (IMUs) based on Micro-Electro-Mechanical System (MEMS) technology have been in the focus of past and current research. In comparison to high-end IMUs, an entire Inertial Navigation System (INS) can be implemented with smaller size/volume, lower weight and costs. In exchange, they have a rather low accuracy performance due to their large systematic errors such as biases, scale factors and drifts, which are strongly dependent on temperature [1]. Hence, MEMS IMUs are not used for traditional, stand-alone, inertial applications but for integrated systems in which they are coupled with other sensors to compensate the temporally increasing errors. Regarding robust navigation, the short-time stable INS is often combined with the longtime stable Global Navigation Satellite System (GNSS). Three different INS/GNSS integration strategies are well known: the loosely, tightly and deeply coupled systems. They differ in their system integration level, their inter-system dependencies, achievable integrity and design complexity. Among these strategies, the loosely coupled system provides the lowest dependency level and simplest system design [2]. Thus, it is widely used in today's integrated applications. For this scheme, the position and velocity estimates of a GNSS receiver are used as additional observations in the INS position filter to enhance the INS accuracy. However, the GNSS PVT (PositionVelocity-Time) solution is not affected by the INS. Considering the case where no GNSS PVT solution is available, i.e. less than four satellites are visible, the INS can exclusively determine the position for a certain period of time. The length of this period depends on the quality of the IMU, i.e., its stability and error performance. Out of the different IMU technologies, MEMS based IMUs have the worst error performance, i.e., high instabilities, drifts and measurement noise. Consequently, they are normally only able to sufficiently coast the position for a couple of seconds.

To improve the inertial error performance, a network of MEMS IMU sensors could be used. By exploring the emerging measurement redundancy with suitable combination strategies and appropriate sensor distributions, the coast time can be significantly increased. Due to the size and price decreasing trend of the sensors, the investigation of sensor networks is a very promising approach with respect to economical and ergonomic consequences. For instant, skew-redundant IMUs (SRIMUs) consist of a redundant number of inertial sensors skewed against each other. Their configuration encapsulates a maximum amount of information depending on the number of sensors and the configuration geometry. Studies have shown that the gained redundancy can improve the INS performance significantly [1], [3], [4], [5]. Via simulations and theoretical derivations, Sukkarieh et. al in [4] have shown that the accuracy can be improved by $33 \%$ by placing the MEMSIMUs on a thetrahedron. Other studies [3] compared the performance difference of orthogonally-redundant and skewredundant IMUs.

In combination with GNSS, it can improve the system performance on several levels. The noise estimation can be obtained directly from the data and more realistic, stochastic error modeling is possible. Consequently, the noise level can be reduced and faulty signals and sensor malfunctioning can be detected and isolated. Furthermore, sensor error calibration is achievable even during uniform motion or static initialization [3]. Based on commonly acknowledged models [3], [6], we simulate a network of identically and orthogonally mounted IMUs and determine their system performance via Monte-Carlo simulations. We show that the accuracy and stability performance can be significantly improved.

In this paper, we investigate the effect of the inertial redundancy in a Ground Based Augmentation System (GBAS) for aviation. In GBAS, local reference receivers located around the airport are used to formulate a correction message, which is transmitted to users. A receiver on an aircraft uses this information to correct GPS signals [7]. In this paper, the corrected GPS position is combined with the inertial network 
and the performance.

Moreover, we apply an integrity concept based on the known GNSS RAIM (Receiver Autonomous Integrity Monitoring) algorithm [8] to inertial sensors. We investigate this integrity algorithm in both, analytically as well as via simulations. Our new system presented in this paper is a low-cost and low-complex approach providing sufficient GNSS outage bridging performance while achieving the required integrity standards.

\section{SYSTEM DESCRIPTION}

\section{A. Inertial Sensor Error Model}

Complex and detailed based on different technologies have been presented in [2] and [1]. According to [6], however it is sufficient to use a simplified error model for low-cost sensors. Given that the misalignment of the different measuring axes are known, it can be written as:

$$
\hat{s}(t)=\left(1+s_{f}\right) s(t)+b(t),
$$

where $\hat{s}(t)$ is the measured sensor output, i.e an angular turn rate and longitudinal acceleration, respectively. The true value of this quantity is denoted as $s(t)$. This true value is corrupted by a certain scale factor $s_{f}$ and a bias $b(t)$. The latter can be modeled by a constant offset $b_{0}$ as well as a time varying component $b_{1}(t)$ and sampling noise $b_{w}$. In this paper, we will assume that the scaling factor and the offset are corrected by an initial calibration of the sensors. Additionally, the sampling noise is assumed to be Gaussian distributed with zero-mean and a given variance $\sigma_{w}^{2}$. Based on several measurement analysis in [6], the time-varying component can be represented by a 1st-order Gauss-Markov process such as

$$
\dot{b}_{1}(t)=-\frac{1}{\tau} b_{1}(t)+n_{b_{1}},
$$

where $\tau$ is the time constant or also called correlation time and $n_{b_{1}}$ is the driving process noise which can be assumed to be Gaussian distributed with zero mean and variance $\sigma_{b_{1}}^{2}$. This error model is applied to the acceleration as well as to the turn rate values in order to simulated a realistic behavior of IMU.

These erroneous inertial measurements are then processed by a traditional strapdown algorithm. Hence, the following navigation equations need to be solved [1]:

$$
\left[\begin{array}{c}
\dot{\boldsymbol{r}}^{n} \\
\dot{\boldsymbol{v}}^{n} \\
\dot{\boldsymbol{R}}_{b}^{n}
\end{array}\right]=\left[\begin{array}{c}
\boldsymbol{v}^{n} \\
\boldsymbol{R}_{b}^{n} \boldsymbol{f}^{n}-\left(\boldsymbol{\omega}_{i n}^{n}+\boldsymbol{\omega}_{i e}^{n}\right) \times \boldsymbol{v}^{n}+\boldsymbol{g}^{n} \\
\boldsymbol{R}_{b}^{n}\left(\boldsymbol{\omega}_{i b}^{b}-\boldsymbol{\omega}_{i n}^{b}\right)
\end{array}\right],
$$

given the observations $\boldsymbol{\omega}_{i b}^{b}$ and $\boldsymbol{f}^{n}$. It is assumed that the gravitation $\boldsymbol{g}^{n}$, and the initial conditions are known.

\section{B. Redundant Inertial Navigation}

Inertial sensor networks have been investigated in literature [3], [4], [5], [9]. Waegli in [3] has compared the performance difference of orthogonally-redundant and skewredundant IMUs. Based on these results, we investigate a low complex approach in order to obtain a first idea of the GBAS performance impact. We use three orthogonally mounted lowcost IMUs. We assume that the misalignment between the IMUs is perfectly known and hence, they are not discussed further. The redundancy is exploited at the sensor level. This means each IMU is processed individually and their combined sensor information is used to obtain the navigation solution. Consequently, the combined measurement noise is reduced by a factor of $\sqrt{3}$ assuming the measurement noise of the different IMUs is independent and shows a constant variance during the measurements [1]. Furthermore, this combined inertial solution based on Equation (3) is used for the loosely-coupled GBAS integration.

\section{INS/GBAS Integrated Navigation}

For our investigations, we consider a loosely coupled INS/GPS integration algorithm. This scheme offers a high interchangeability of the involved GNSS and IMU sensors. Based on the nature of the inertial navigation, the pure inertial navigation error increases over time since it accumulates all occurring measuring errors. Consequently, the INS needs a frequent update of its error behavior in order to compensate these errors.

In this investigations, the GBAS based GPS navigation solution is used to obtain these errors. That is a full satellite based solution is required to correct inertial position errors. In case no GPS based solution is available, the INS starts to drift and the position error increases. The degradation of the INS over time depends on the quality of the IMU itself. Given a certain maximum error performance, the time of GPS outages coasted by the INS is limited. In this paper, we use a redundant network of inertial sensors to increase the performance of low-cost MEMS sensor while maintaining low cost and low complexity.

\section{Ground Based Augmented System}

Ground Based Augmentation Systems (GBAS) have been developed to allow robust local navigation for precision approach of aircraft with $2 \mathrm{~Hz}$ position update. The GBAS architecture has been investigated the last 25 years [10]. In 2009, a first GBAS station was approved for CAT I precision approach procedures. The current work is concentrated in the development of GBAS for CAT III precision approaches. Two different architectures are proposed:

- A short term one, keeping the actual constellation (GPS single frequency configuration) and proposing additional monitors on ground and on board to achieve the stringent integrity and continuity requirements

- A long term one considering a multi-constellation (GPS and Galileo), multi frequency (L1/E1, L5/E5a, E5b) based GBAS solution

The user corrects its pseudo-range measurements using the pseudo range corrections and the range rate corrections received from the ground subsystem through the VHF Data Broadcast (VDB) link. Simultaneously, the user calculates the integrity information of the position in terms of protection levels for the fault free case $\left(H_{0}\right)$ and for the reference receiver 
fault case $\left(H_{1}\right)$. The resulting protection level is the maximum of the two. For Differentially Corrected Positioning Service (DCPS) an additional protection level the so called ephemeris protection level is considered. It accounts for large ephemeris error observed by the ground subsystem for a given satellite. The overall protection level is then the maximum of the three.

\section{INTEGRITY CONCEPT}

\section{A. Protection level concept of an inertial based navigation solution}

The short term stability of MEMS based INS is compensated by an accurate GBAS solution. Protection levels as defined in the Minimum Operational Performance Standards (GBAS MOPS) should be extended to take into account the contribution of the inertial navigation system. The Vertical Protection Level (VPL) expression of the combined solution depends on the vertical protection level of each system as well as the used integration scheme. For simplification, only the VPL is considered in this chapter. Similarly considerations can be done for the Lateral Protection Level (LPL) or the Horizontal Protection Level (HPL).

We recall the fault free vertical protection level for a GBAS system (for more details see for example [7]):

$$
\mathrm{VPL}_{\mathrm{GBAS}, H_{0}}(t)=K_{\mathrm{ffmd}} \sigma_{\mathrm{GBAS}, \mathrm{V}}
$$

with $K_{\text {ffmd }}$ representing a multiplier which determines the probability of fault-free missed detection and $\sigma_{\mathrm{GBAS}, V}$ is the standard deviation of the over-bounded vertical error of a GBAS solution. This protection level can be estimated at each time step depending on the ground subsystem information and the actual geometry of satellites chosen to calculate the position.

The inertial navigation system is based on longitudinal and rotational acceleration sensors. The preceding chapter proposed a sensor error model which is a random model taking into account sensor imperfection. Thus the navigation solution error is a random process solution of a stochastic differential equation (see Equation (3)). Assuming the random process is a diffusion process, it can be characterized by a drift and a diffusion coefficient.

We define a protection level for the navigation solution using a stand-alone INS only for a short time period from 0 to $T$ :

$$
\operatorname{VPL}_{\mathrm{INS}}(T)=\max _{0 \leq t \leq T}\left(\left|b_{\mathrm{INS}, V}(t)\right|\right)+K_{\mathrm{ffmd}} \max _{0 \leq t \leq T}\left(\sigma_{\mathrm{INS}, V}\right),
$$

where $\max _{0<t<T}\left(\left|b_{\text {INS }, V}(t)\right|\right)$ is the maximal absolute bias of the INS in the the vertical direction during the time period $[0, T]$ and $\sigma_{\mathrm{INS}, V}$ is the standard deviation of the overbounded vertical component of the INS error. This standard deviation is a function of time. The same applies for the bias, therefore it is necessary to take the maximum value during the time period $[0, T]$.

1) Theoretical vertical protection level using a simplified scenario: Let consider only a vertical acceleration of the aircraft without any attitude change. Under these conditions, the vertical position of the aircraft can be determined using only the vertical accelerometer or the combination of redundant vertical accelerometers. We simplify the Equation (1) to obtain:

$$
\hat{s}(t)=s(t)+b_{1}(t),
$$

given that the scaling factors $s_{f}$, null-shifts $b_{0}$ and the sampling noise $b_{w}$ are zero. the time-varying bias $b_{1}(t)$ is solution of the stochastic differential Equation (2).

If we rewrite the equation 2 in a differential way and introducing the one-dimensional Brownian motion $B_{t}$ we obtain:

$$
d b_{1}=-\frac{1}{\tau} b_{1} d t+\sigma_{b 1} d B_{t}
$$

It can be seen that under the considered assumptions $b_{1}$ is an Itô diffusion process characterized by a drift $-\frac{1}{\tau} b_{1}$ and a diffusion coefficient of $\sigma_{b_{1}}^{2} / 2$.

We are interested in the evolution of the vertical position with respect to time therefore the only variables considered are the time and the vertical component of the position $x_{v}$. The generator of this diffusion process can be expressed by:

$$
A f(x)=-\frac{1}{\tau} b_{1} \frac{\partial f}{\partial x_{v}}+\frac{1}{2} \sigma_{b_{1}}^{2} \frac{\partial^{2} f}{\partial x_{v}^{2}}
$$

for any $f \in C_{0}^{2}(\mathbb{R})$.

The Kolmogorov Forward Equation gives the law of evolution of the density of distribution of the parameter $b_{1}$

$$
\begin{gathered}
\frac{\partial p_{b_{1}}}{\partial t}=\frac{1}{\tau} \frac{\partial b_{1} p_{b_{1}}}{\partial x_{v}}+\frac{1}{2} \sigma_{b_{1}}^{2} \frac{\partial^{2} p_{b_{1}}}{\partial x_{v}^{2}} \\
p_{b_{1}}\left(0, x_{v}\right)=f\left(x_{v}\right)
\end{gathered}
$$

where $\mathrm{f}$ is the initial density of $b_{1}$.

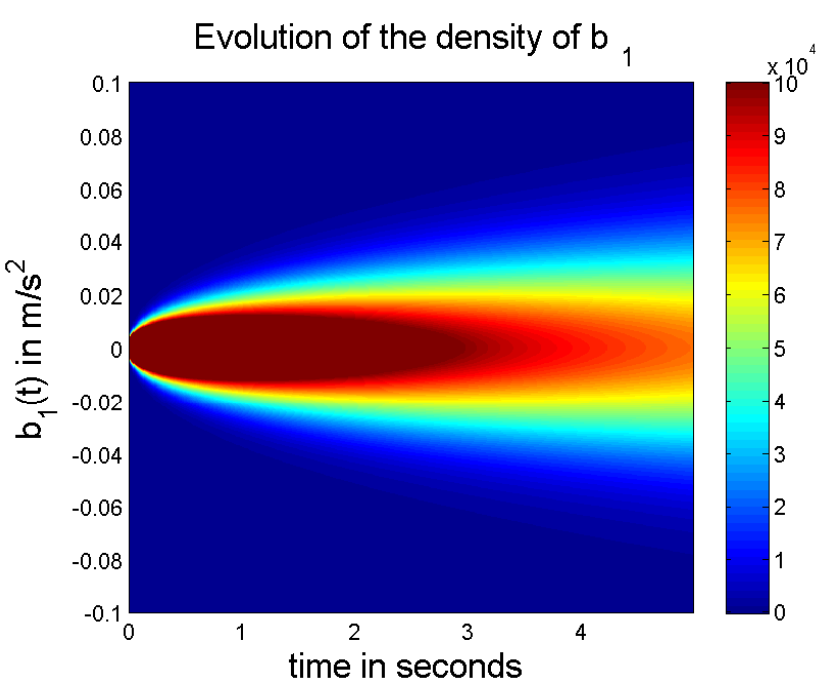

Fig. 1. Evolution of the density of $b_{1}$ vs. $\mathrm{t}$ for $\tau=100$ seconds and $\sigma_{b_{1}}=1.2$ milli g

Figure 1 shows the solution of the Kolmogorov forward equation. The integration time is 5 seconds. The used param- 
eters are $\tau=100$ seconds and $\sigma_{b_{1}}=1.2$ milli-g as proposed in [6]. We assumed an initial Gaussian distribution of $b_{1}$ to be centered with a standard deviation of $10^{-3} \mathrm{~m} / \mathrm{s}^{2}$.

To show the impact of imperfections in the accelerometer, let us assume the true acceleration to be zero. The uncertainty in the acceleration due to imperfections in the accelerometer are defined as:

$$
\hat{s}(t)=b_{1}(t)
$$

$\hat{s}(t)$ is a random process following the same law as $b_{1}(t)$. The impact of these imperfections in velocity and in position domain can be obtained by simple integration with respect to time. Assuming the vertical speed at $t=0, v_{v}(0)=v_{0 v}$ and the initial height $h(0)=h_{0}$, Figure 2 shows the resulting altitude error density. In the simulations, we considered the initial vertical speed to be zero $\left(v_{0 v}=0\right)$.

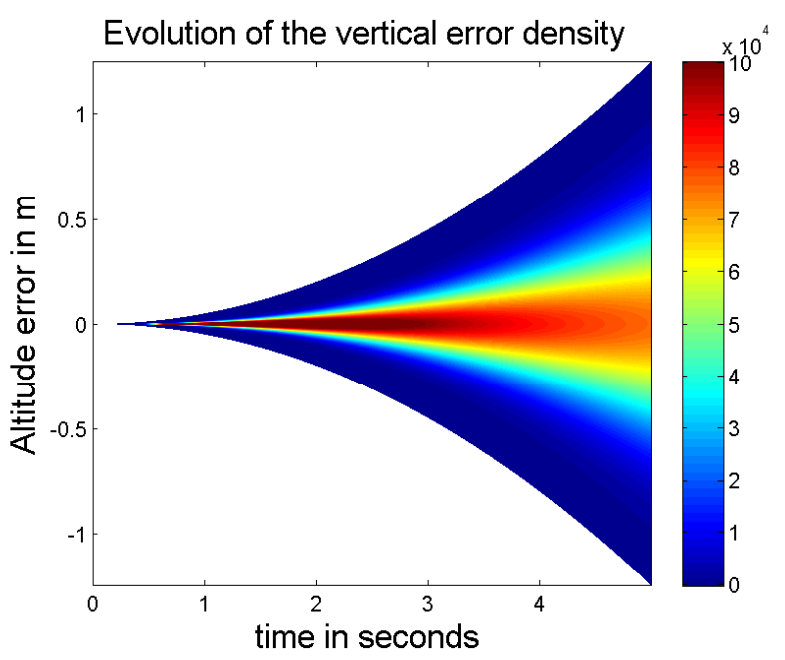

Fig. 2. Evolution of the density of altitude error vs. $\mathrm{t}$ for $\tau=100$ seconds and $\sigma_{b_{1}}=1.2$ milli $\mathrm{g}$

For each time $t$, it is possible to find the Gaussian over bound of the vertical error, take the maximum standard deviation of all these Gaussian over bounds in the considered period of time and multiply it with $K_{\text {ffmd }}$ to find the impact of the accelerometer imperfections in the protection levels. As the altitude error is a diffusion process, the maximum values are obtained at $t=T$ (at the end of the time period). We can see that the protection level is very sensitive to the integration time. We considered the initial distribution of $b_{1}$ to be centered which is not the reality. A non centrality term will automatically introduce a bias in the altitude error and should be taken into consideration in the protection level equation (5).

2) Impact of the use of 2 redundant accelerometers: Considering the usage of 2 redundant accelerometers with independent imperfections, the measurement given by the accelerometer 1 and 2 is defined as $\hat{s}_{1}(t)$ and $\hat{s}_{2}(t)$, respectively. The average of these observations at each time $t$ as the best estimate of the acceleration in the vertical direction [1].

$$
\hat{s}(t)=\frac{1}{2}\left(\hat{s}_{1}(t)+\hat{s}_{2}(t)\right) .
$$

The stochastic differential equation verified by $b_{1 i}$ for the accelerometer $i$ is:

$$
d b_{1 i}=-\frac{1}{\tau} b_{1 i} d t+\sigma_{b 1 i} d B i_{t}
$$

Considering the simplified Equation (11),

$$
\hat{s}(t)=\frac{1}{2}\left(b_{11}(t)+b_{12}(t)\right) .
$$

Assuming two independent Brownian motions with the same diffusion coefficients:

$$
d \hat{s}=-\frac{1}{\tau} \hat{s} d t+\frac{1}{2}\left(\begin{array}{ll}
\sigma_{b 1} & \sigma_{b 1}
\end{array}\right)\left(\begin{array}{c}
d B 1 \\
d B 2
\end{array}\right),
$$

the drift coefficient is the average of accelerometer drifts and the diffusion coefficient is $\sigma_{b_{1}}^{2} / 4$, which is half of the diffusion coefficient of an accelerometer alone.

In the case where both accelerometers have the same drift, the combination will remain after averaging (no benefit of redundancy). If the drifts are equal in magnitude but opposite in sign, the averaging will eliminate the drift (full benefit of redundancy). These properties directly impact the protection levels for the inertial system by keeping or reducing the bias term of equation 5 and reducing by a half the component due to the diffusion part.

\section{B. Integrity concept of INS/GBAS integrated solution}

The GBAS system is designed for precision approach under low visibility conditions. The integrity and continuity requirements are therefore very stringent. Inertial aiding provides additional robustness. As mentioned in one of the previous sections, we consider a low-complex INS/GBAS integration scheme which calibrates the INS with GBAS when GBAS is available (in a nominal operation every 0.5 second). That is the vertical position error density generated by the GBAS system will be the initial condition for the density of the INS error during the coasting phase $(n / 2<t<(n+1) / 2)$.

Figure 2 shows the density of the vertical error with the Dirac density at $t=0$. In order to consider the effect of a vertical error due to GBAS, one must calculate for each time step the convolution of the error density due to the imperfection of the sensor with the error density over bound due to the GBAS system (which is considered as a constant until the next update).

\section{Simulation Results}

In the following section, we compare our simulation results of the GBAS integration with the single IMU and orthogonally-redundant INS in terms of position error performance, GBAS outage coasting ability and resulting integrity capability. The GBAS related data has been recorded during a flight trail with the DLR's experimental GBAS facility in Braunschweig on November, 27th in 2009. The inertial data is 


\begin{tabular}{|c|c|c|c|}
\hline Sensor & $\sigma_{w}$ & $\sigma_{b_{1}}$ & $\tau$ \\
\hline \hline Gyro & $0.05\left[{ }^{\circ} / \mathrm{hr}\right]$ & $360\left[{ }^{\circ} / \mathrm{sec}\right]$ & $300[\mathrm{sec}]$ \\
Acceler. & $1 \times 10^{-3}[\mathrm{~g}]$ & $2.4 \times 10^{-3}[\mathrm{~g}]$ & $100[\mathrm{sec}]$ \\
\hline
\end{tabular}

TABLE I

SimUlation PARAMETERS OF THE GYROSCOPE AND ACCERLEROMETER ERROR MODELS GIVEN IN [6], WHERE $\sigma_{w}^{2}$ IS THE SAMPLING NOISE VARIANCE, $\sigma_{b}^{2}$ IS THE DRIVING NOISE VARIANCE OF THE 1 ST-ORDER GAUSS-MARKOV PROCESS AND $\tau$ ITS CORRELATION TIME.

simulated accordingly to the previously discussed error model. The used model parameters can be seen in Table IV.

We consider two different scenarios, first GBAS is available throughout the flight trail and secondly synthetic GBAS or rather GPS outages occur with a duration of $\Delta t$ seconds.

\section{A. Position Accuracy}

Investigating the two different INS schemes, the orthogonally-redundant IMU clearly outperforms the single IMU approach. That is the former produces on average a twice better error performance than the latter one. In Figure 3 the average position improvement of the two stand-alone INS over time is displayed. It can be seen that the beginning the improvement is much higher than in the end. This is due to the long-term error characteristics of the IMUs. Consequently, the most performance improvement of the redundant IMU in comparison to the single IMU can be expected for short-time GPS outages.

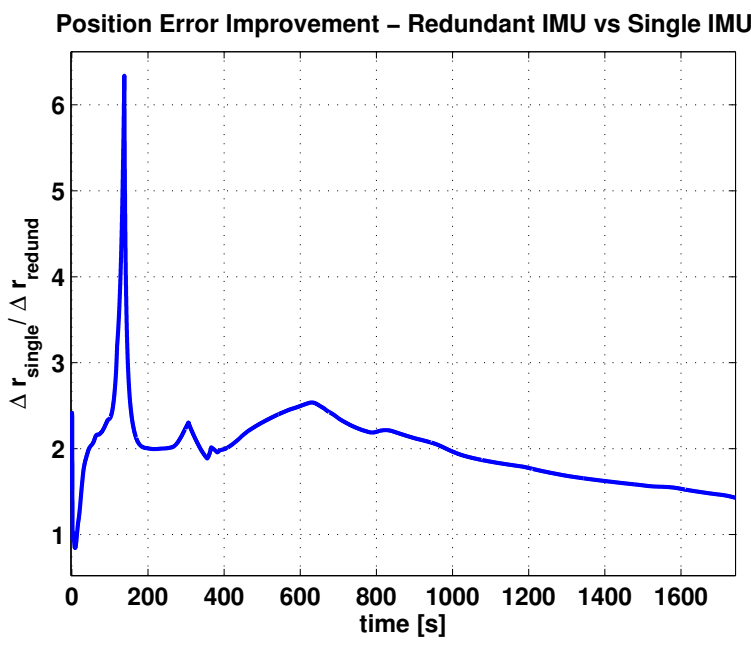

Fig. 3. Position Error performance improvement of the three orthogonallyredundant IMUs in comparison to the single IMU case.

\section{B. Coasting of GBAS outages}

Based on the previous results, it can be expected that the redundant IMU/GBAS scheme allow much larger GPS outage durations. In Figure 4 the error performance of the redundant IMU/GBAS for different GBAS outage durations $\Delta t$ is displayed. The longer this duration is the larger is the error. The error improvement factor to the single IMU case is shown in Figure 5.

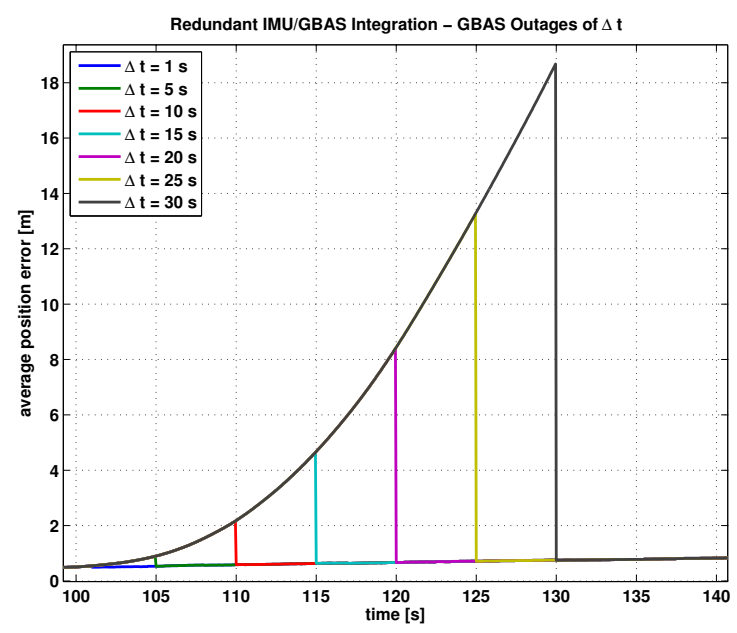

Fig. 4. Position error performance for different GBAS outage durations

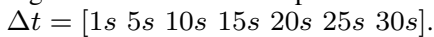

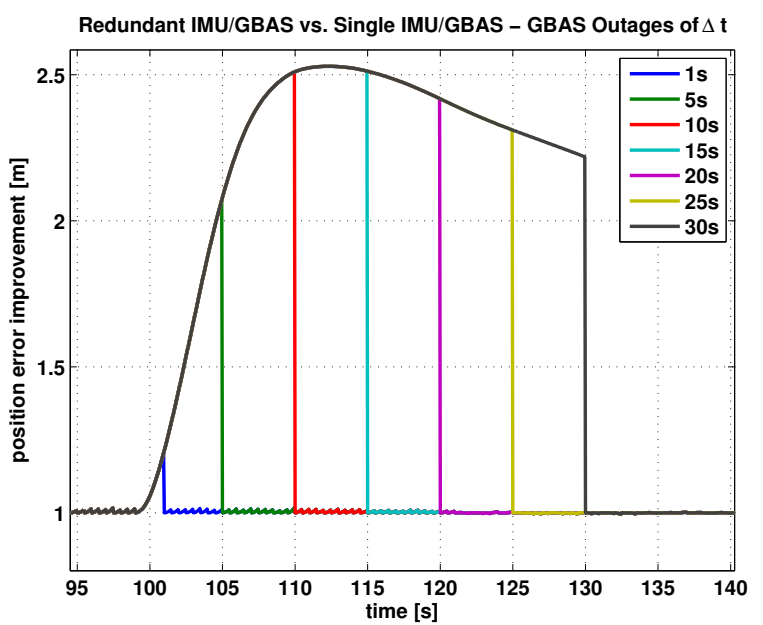

Fig. 5. Position error performance improvement for different GBAS outage

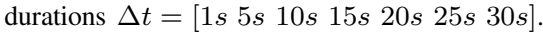

\section{Integrity Analysis}

Comparison of the integrity information to the alert levels defined for civil aviation. The alert levels define a maximum allowed error performance for CAT I precision approach. It can be seen, that the protection levels based on the redundant IMU/GBAS scheme full the requirements for a maximum outage duration of $10 \mathrm{~s}$, where as the single IMU/GBAS scheme only allows a maximum duration of $5 \mathrm{~s}$. This can be seen in Figure 6 and Figure 7, respectively.

\section{CONCLUSION}

In this paper, we have shown that three orthogonally mounted low-cost MEMS-IMUs can improve the performance of an INS/GBAS scheme by the factor of two. This preliminary investigations with a relative simple integration approach illustrate the potential of redundant IMUs for integrated systems. We further introduced and discussed a low-complex integrity 


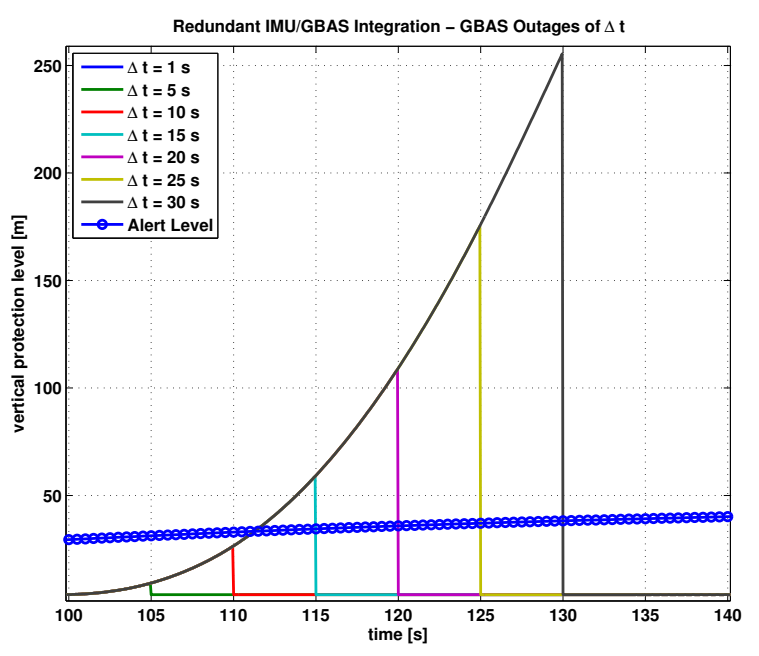

Fig. 6. Protection level performance of the redundant IMU/GBAS scheme

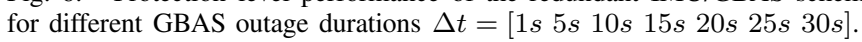

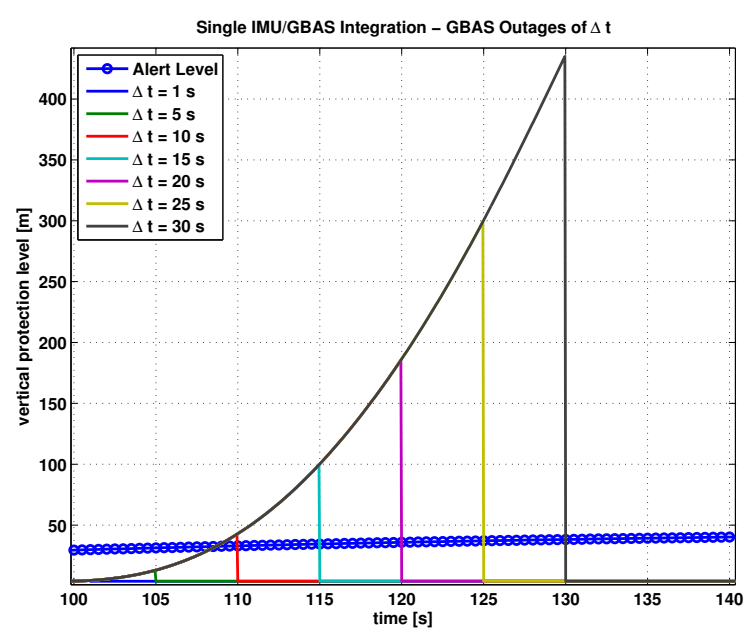

Fig. 7. Protection level performance of the redundant IMU/GBAS scheme

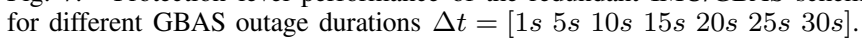

concept to access integrity information even during GBAS outages. This capability is essential for all safety-of-life applications, especially in civil aviation.

\section{REFERENCES}

[1] D. H. Titterton and J. L. Weston, Strapdown Inertial Navigation Technology, 2nd Edition, D. N. Stewart and P. H. Griffiths, Eds. The Institution of Electrical Engineers, 2004.

[2] J. Wendel, Integrierte Navigationssysteme, Sensordatenfusion, GPS and Inertiale Navigation. Oldenbourg Verlag München Wien, 2007.

[3] A. Waegli, S. Guerrier, and J. Skaloud, "Redundant mems-imu integrated gps for performance assessment in sports," I. PLANS, Ed., Monterey, California, May 5-8 2008

[4] S. Sukkarieh, P. Gibbens, B. Brocholsky, K. Willis, and H. DurrantWhyte, "A low-cost redundant inertial measurement unit for unmanned air vehicles," The International Journal of Robotics Research, vol. 19, p. $1089,2000$.

[5] A. Waegli, "Trajetory determination and analysis in sports by satellite and inertial navigation," Ph.D. dissertation, Ècole Polytechnique Fédérale de Lausanne, 2009.
[6] D. Gebre-Egziabher, "Design and performance analysis of a low-cost aided dead reckoning navigator,' Ph.D. dissertation, Stanford University, 2004.

[7] RTCA/SC-159, "RTCA/DO-245A: Minimum Aviation System Performance Standards for the Local Area Augmentation System (LAAS)," RTCA, Tech. Rep., 2004.

[8] T. Walter and P. Enge, "Weighted raim for precision approach," in Proceedins of the ION GPS. Institute of Navigation, 1995, pp. 1995 2004.

[9] A. Waegli, J. Skaloud, P. Tomé, and J.-M. Bonnaz, "Assessment of the integration strategy between gps and body-worn mems sensors with application to sports," I. GNSS, Ed., Fort Worth, Texas, USA, 2007.

[10] T. Murphy, "Ground Based Regional Augmentation Systems Architectures and Performance," October 2000. 